\title{
Reactive oxygen species induced oxidative stress, neuronal apoptosis and alternative death pathways
}

\author{
Kandaswamy Selvakumar1, Gunasekaran Krishnamoorthy ${ }^{2}$, Prabhu Venkataraman ${ }^{3}$, \\ Jagadeesan Arunakaran $^{1 \#}$ \\ ${ }^{1}$ Department of Endocrinology, Dr. ALM Post Graduate Institute of Basic Medical Sciences, University of Madras, Chennai, India \\ ${ }^{2}$ Department of Biochemistry, Asan Memorial Dental College \& Hospital, Asan Nagar, India \\ ${ }^{3}$ Department of Medical Research, SRM Medical College, Kattunkulathur, India \\ Email: \#_arunakaran@hotmail.com
}

Received 15 October 2012; revised 23 November 2012; accepted 7 January 2013

\begin{abstract}
Reactive oxygen species (ROS) are produced as a byproduct of cellular metabolic pathways and function as a critical second messenger in a variety of intracellular signaling pathways. The excessive intracellular generation of ROS on the other renders a cell oxidatively stressed. This involvement of ROS in numerous diseases has been documented and at different phases of the apoptotic pathway such as induction of mitochondrial permeability transition and release of mitochondrial death amplification factors, activetion of intracellular caspases and DNA damage has been clearly established. Cell death by apoptosis is a part of normal development and maintenance of tissue homeostasis. Polychlorinated biphenyls, one of the environmental pollutants which are widely used in electrical industries and lipophilic and resistant to biological decomposition accumulate through food chain. They are developmental neurotoxicants which induce neuronal apoptosis. Our studies proved that oxidative stress is induced promoting LPO and a decrease in all the antioxidant enzymes in testis, epididymis, ventral prostate, seminal vesicles, liver, kidney and brain regions. Neuronal damages were observed in all the brain regions after PCB exposure. PCB increased caspase8 mRNA/protein expression in hippocampus of adult rats. This upregulation results in Fas-FasL mediated induction of hippocampal apoptosis. Performin/granzyme induced apoptosis is the main pathway used by cytotoxic lymphocytes to eliminate virus-infected or transformed cells. The production of ROS is greatly increased during reperfusion phase when oxygen becomes available and the mitochondrial respiratory chain is impaired. Furthermore, this is exacerbated by reduced antioxidant defenses.
\end{abstract}

\footnotetext{
"Conflict of interest: no.

\#Corresponding author.
}

Keywords: Apoptosis; Caspases; Cell Death; Mitochondria; PCBs; Reactive Oxygen Species

\section{INTRODUCTION}

Cellular apoptosis is a complex process that is triggered by extrinsic and intrinsic signals. The extrinsic (external) pathways involves the activation of a death receptor upon binding of its ligand, recruitment of specific proteins at the death domain and downstream signaling through a cascade of protein-protein interactions. The intrinsic pathway involves the mitochondria and the release of proapoptotic factors into the cytosol with subsequent activation of executioner caspases.

\section{APOPTOSIS}

Apoptosis is a programmed cell death. Many physiological and developmental stimuli cause apoptosis and the mechanism is used frequently to delete unwanted superfluous or potentially harmful cells such as those undergoing transformation. It involves cell shrinkage; condensation of chromatin in the periphery of the nucleus, cell membrane blebbing and DNA fragmentation. Eventually, the cell breaks up into many membrane bound apoptotic bodies which are phagocytized by neighbour cells.

\section{DEATH RECEPTORS}

Death receptors belong to the tumor necrosis factor (TNF)/nerve growth factor receptor superfamily and are transmembrane proteins with three domains: an extracellular ligand binding domain, a transmembrane domain and a terminal domain. Death receptors are involved in transmitting the death signal from the cell surface to intracellular pathway upon substrate binding [1]. The best characterized death receptors which functions in apoptosis are Fas, TNF receptor 1 (TNFR1), TNF related apoptosis including ligand receptor 1 (TRAIL-R1) (death 
receptor 4, DR4) and TRAIL receptor 2 (TRAIL-R2) death receptor 5 (DR5). A soluble Fas decoy receptor De R3 and three decoys of TRAIL receptors, TRAIL R3, TRAIL R4 and osteoprotegrin (OPG) have been characterized but they are unable to trigger apoptosis because of the absence or truncation of the cytoplasmic death domain. The ligands that bind to the death receptors belong to the TNF superfamily of cytokines, which is comprised of TNF- $\alpha$, FasL and TRAIL. The first step in receptor-mediated apoptosis is initiated by ligand binding to the death receptor, a process that causes receptor trimerisation and recruitment of adaptor proteins at the cytosolic death domain (DD) of the receptor. Fas and TRAIL-associated death domain (FADD/TRADD) proteins further bind procaspases- 8 and/or -10 and form the DISC formation and strong activation of caspase- 8 that directly activates the downstream executioner caspases-3 and -7 .

In type II cells, DISC formation is weak and therefore insufficient to induce direct caspase- 8 catalyzed apoptosis per se, rather it initiates the cleavage of a proapototic protein, Bid and that in turn engages mitochondria apoptotic signaling. Thus, the mitochondria functions as amplifiers that induce the activation of executioner caspases. Type II cells strongly depend on caspase-9 activation and the mitochondrial amplification loop to mediate Fas induced apoptosis. This finding provides evidence for the two pathway model viz. the extrinsic death receptor and the intrinsic mitochondrial pathways of cell apoptosis mediated by death receptors.

The death receptor pathway has been best described in the immune system. Activated T cells and Nature Killer (NK) cells are the major cells expressing FasL and the mutations in Fas/FasL in humans lead to the complicated immune disorder, autoimmune lympho-proliferative syndrome. Normally, the Fas-FasL is weakly expressed in CNS, including glial cells and neurons and is associated with the maintenance of immune suppression and prevention of an inflammatory response.

Fas presence in developing brain was involved in neurite remodeling. Fas mediate neuronal branching through its death domain, without caspase activation. Under condition of stroke, an increased expression of Fas/FasL could induce neuronal apoptosis and contribute to neurodegeneration. Thus it appears that the Fas system within the CNS has a dual function namely the participation in neurite branching during development and in neuronal apoptosis during the disease state. In pathological conditions, elevated TNF- $\alpha$ and TNF- $\alpha$ receptors expression were shown to contribute to neuronal cell death [2].

\section{MITOCHONDRIAL PATHWAY}

Mitochondria are key players in the early induction and regulation of apoptotic cell death. Apoptotic stimuli such as DNA damage, ROS, or Fas signaling mediated mitochondrial cell death by a process that results in the release of small pro-apoptotic proteins that are normally located in the mitochondrial inner membrane space (Figure 1). Once in the cytosol, pro-apoptotic proteins like cytochrome $\mathrm{C}$, second mitochondria-derived activator of caspases/direct IAP binding protein of low pI AIF and endonuclease $\mathrm{G}$ trigger caspase dependent or independent apoptotic death pathways. In the caspase-dependent mechanism, cytochrome $\mathrm{C}$ binds to an adaptor molecule, apoptotic protease activating factor 1 (Apaf-1) to form the apoptosome where procaspase- 9 is recruited and activated in the presence of ATP. Caspase- 9 further cleaves and activated the effector caspases, procaspase- 3 and/or -7, which process substrates like caspase activated DNase (ICAD) or PARP, and leads to DNA fragmentation. In a caspase-independent manner, AIF translocates to the nucleus where it induces DNA fragmentation and chromatin condensation while endo $G$ induces internucleosomal DNA fragmentation.

\section{POLYCHLORINATED BIPHENYLS (PCBs) INDUCED OXIDATIVE STRESS}

PCBs are a family of halogenated aromatic hydrocarbons and one of the environmental pollutants, which are widely used in electrical industries [3]. They are lipophilic and resistant to biologic decomposition and can accumulate in higher tropic levels through the food chain [4]. Aroclor 1254 is a commercial mixture of PCBs. PCBs are developmental neurotoxicants and it has been shown to produce neurochemical alterations in several experimental settings [5] as well as behavioral changes in learning, memory, motor activity and sexual behavior [6]. PCBs especially are highly chlorinated. PCBs may selectively induce cytochrome $\mathrm{P} 450 \mathrm{~s}$ as a possible source of ROS or alternatively the oxidation of a broad range of endogenous and exogenous substances. Oxidative stresses induced by PCBs are due to interaction of these compounds with aryl hydrocarbon receptors (AhR) (Figure 1) and activation of the cytochrome P450 1A superfamily. The cytochrome P450 catalyzed oxidation of lower chlorinated biphenyls gives rise to mono and di hydroxyl metabolites. Later can auto oxidize or be enzymatically oxidized to semi-quinone and /or quinines [7]. Some PCBs quinones undergo redox cycling with the formation of ROS thus becoming another source of oxidative stress [9]. ROS like $\mathrm{O}_{2}^{-}, \mathrm{HO}$ and $\mathrm{H}_{2} \mathrm{O}_{2}$ are thought to contribute to LPO, DNA damage and protein degradation.

Reactive oxygen species are closely involved in several diseases of nervous system including Parkinson's disease, Schizophrenia and Alzhemier's disease. ROS initiated oxidative stress can be regulated by cell defense 


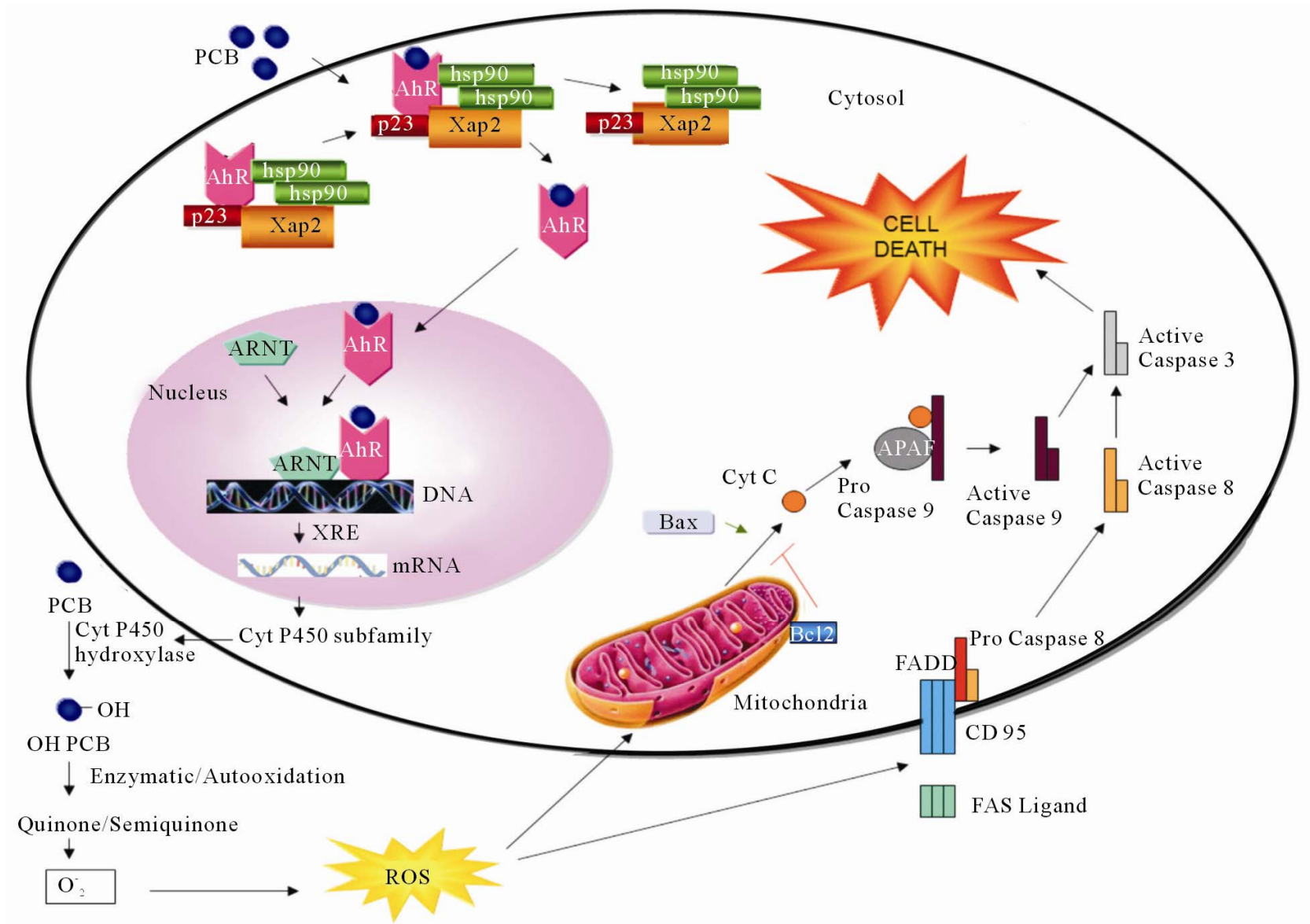

Figure 1. ROS induced apoptosis. Schematic diagram of PCB influences ROS induced apoptosis by AhR mechanism. Mechanisms of AhR and apoptosis were adopted and combined from Pravettoni et al., [7] and Engel and Evens [8], respectively.

mechanisms which include enzymatic antioxidants such as SOD, GPx [10]. The inherent biochemical and physiological characteristics of the brain including high poly unsaturated fatty acids and energy recruitments make it particularly susceptible to free radicals mediated insult [11].

Our findings reveal that PCB reduces fertility in male rats by reducing the organ weight of accessory sex organs, serum testosterone, estradiol, thyroid hormone profiles, testicular Leydig and Sertoli cellular function as well as histoarchitecture. It also induces oxidative stress in sperm parameters. PCB exposure decreased steroidogenic machinery through decreasing testicular Leydig cellular steroidogenic enzymes such as $3 \beta \mathrm{HSD}, 17 \beta \mathrm{HSD}$. Oxidative stress is induced by promoting lipid peroxidation, and a decrease in the activity of antioxidant enzymes in testis, epididymis, ventral prostate, seminal vesicles, liver, kidney and brain regions [12-17]. Recently we have studied that mRNA expression of $\mathrm{Cu}$ / $\mathrm{Zn}$ SOD and GPx were decreased in most of the brain regions of PCB exposed rats [16]. Neuronal damages were also observed in all the brain regions after PCB exposure.
Cell death by apoptosis is a part of normal development and maintenance of testicular homeostasis. During various stages of spermatogenesis an adequate amount of germ cells are eliminated via the process of apoptosis in order to maintain a precise germ cell population in compliance with the supportive capacity of Sertoli cells. The two divergent pathways of testicular apoptosis namely, the receptor mediated Fas/FasL pathway and the mitochondria mediated pathway play a important role in maintaining the germ cell population [18]. In appropriate activation of apoptosis could enormously jeopardize spermatogenesis and hence fertility. ROS is considered a potential signal for apoptosis. Elevated levels of ROS can cause oxidation of the mitochondrial pores thereby disrupting the mitochondrial membrane potential and releasing cytochrome $\mathrm{C}$ thereby activating the mitochondrial mediated pathway of apoptosis. In addition; ROS have been shown to induce the expression of Fas receptor and ligand stimulating the Fas/FasL-mediated apoptotic signal transduction pathway. Several environmental disruptors are known to inappropriately increase the levels of ROS [19-21]. 
The Fas system has been widely recognized as a key regulator of the apoptosis signal transduction pathways. Fas is a type I transmembrane receptor protein and Fas ligand is a type II transmembrane protein. The ligation of FasL to Fas triggers an FasL mediated apoptotic death in a target cell leading to the activation of caspase 3 [22,23]. All the cells expressing both Fas and FasL do not undergo apoptosis under normal conditions but when an external stimuli is induced then can become significantly sensitized to the Fas mediated apoptosis [24].

Our recent study [21] revealed an evident induction of both Fas and FasL levels in testicular Sertoli cells after PCB treatment. This result was in accordance to onset of FasL mRNA upregulation after Sertoli cell injury [19]. The result indicated that increased expression of FasL in Sertoli cells triggers apoptotic elimination of Fas expressing germ cells, suggests that the upregulation of FasL expression in Sertoli cells and increased activation of caspase 3 may be associated with apoptosis in PCB treated rats.

Co localization of caspase 3 and Fas showed an increased expression of caspase 3 and Fas. The ligation of Fas to FasL could stimulate nuclear factor kappa B (NFkB) activation [24]. NFkB is a complex of p65 and p50 proteins and is found to be constitutively expressed in the nuclei of rat testicular cells. In normal cells, NFkB dimmers are sequestered in the cytoplasm by inhibitory kappa B (IKB). When it exposed to external signal degradation of IKB, free NFkB translocates to the nucleus. NFkB exerts both pro and anti-apoptotic effects in testis upon toxicants exposure [25]. Our study demonstrates that PCB exposure decreased the mRNA expression of NFKB of testicular Sertoli cells [21].

Both intrinsic and extrinsic pathways of apoptosis enter into a common downstream effector caspase 3 which can induce DNA fragmentation by the activation of endonuclease. Cell death by apoptosis is a relatively rapid mechanism and complete removal of the cell often occurs within hours from the tissue [26]. This has been confirmed by histological studies.

\section{PCBS INDUCED NEURONAL APOPTOSIS: HIPPOCAMPUS}

PCBs exposure produces neurodegeneration and oxidative stress. Epidemiological data indicate that PCBs negatively impact neuropsychological function in exposed children [9] and experimental animal studies confirm that developmental exposure to PCBs causes cognitive and psychomotor deficits [27]. These functional deficits reflect subtle perturbations of neuronal connectivity in the developing brain. A critical determinant of neuronal connectivity is apoptosis which is essential to normal brain development occurring in proliferative zones and in post mitotic cells in both the fetal and postnatal brain [28]. Apoptotic signaling pathways are triggered by various molecular cues, one of the most common being ROS. PCBs induced ROS may be linked to increased neuronal apoptosis which was evidenced in primary neuronal cell cultures [29] and this pro apoptotic effect can be blocked by agents that decrease intracellular levels of ROS [30].

Kass and Orrenius [31] demonstrated that a number of environmental toxicants target calcium signaling process, alter them and induce cell death by apoptosis. PCBs cause alterations in cognitive functions as learning and memory in animal models and data are consistent with those obtained in humans [32]. The central components of apoptotic pathways are the proteases of the caspase family. Caspase 3 is a potent effector of apoptosis which triggers via several different pathways in a variety of mammalian cell types and is one of the most important caspases activated downstream of cytochrome $\mathrm{c}$ in the cytochrome $\mathrm{c}$ dependent apoptotic pathways. The increase in the caspase- 3 activity in the hippocampus of PCBs-exposed rats correlates with a decrease in procaspase- 3 content observed by western blot analysis [17].

PCBs influence ROS. ROS induces apoptosis. The mechanism of PCBs on the biological cell is through aryl hydrocarbon receptor mechanism through cytochrome p450 subfamily. PCBs are metabolised through enzymatic/autooxidation and they form quinine or semiquinone. They release superoxide ions. ROS can be derived from exogenous sources or produced in vivo; these include superoxide anion, the hydroxyl radical and hydrogen peroxide. ROS at low levels participates in cell signaling while higher levels are deleterious due to oxidation of proteins; lipids and DNA. Persistent ROS production compromises the cellular antioxidant defense systems and results in oxidative stress and apoptosis.

The schematic representation in the Figure 1 shows the main molecular pathways leading to apoptosis. In the extrinsic pathway upon ligand binding to specific receptors the DISC complex is formed and caspase 8 activated. In the intrinsic pathway, release of cytochrome $\mathrm{c}$ from the mitochondria results in the formation of the apoptosome and activation of caspases 9 and 8, then activate downstream caspase such as caspase 3 resulting in cell death. These two pathways are connected through the cleavage of $\mathrm{BH} 3$ only protein BID.

The extrinsic pathway is induced by ligand mediated activation of tumor necrosis factor family of the cell surface receptors. The most common pathways of proapoptosis, the intrinsic pathway is activated within the cell itself such as in response to stress initiated by damage inducing agents like PCB. In the present study, $\mathrm{PCB}$ induced apoptosis evidenced through the intrinsic 
pathway which involves the release of cytochrome c from mitochondria and is regulated by the Bcl 2 family of proteins upstream of caspase activation [33]. Bcl2 is an apoptosis suppressing factor that hetero dimerizes with Bax and neutralize the effect of the latter. When Bcl2 is present in excess, cells are protected against apoptosis. When Bax is excess and the homodimers of Bax dominate, cells are susceptible to programmed cell death. Therefore, it is the ratio of Bax to $\mathrm{Bcl} 2$ which determines the fate of a cell [34]. Our earlier recent studies demonstrated that PCB treatment showed a significant increase in the activation of caspase 3 followed by an intrinsic pathway of apoptosis in the hippocampus of PCB treated rats [17].

Death receptors are cell surface receptors that transmit apoptotic signals initiated by specific ligands such as Fas ligand, TNF alpha and TNF related apoptosis inducing ligand (TRAIL) which activate finally caspase cascade. Activation of caspase 8 illustrates the TNF receptor signaling pathway. PCB increased caspase 8 mRNA expression in the hippocampus of adult rats. This upregulation results in Fas-Fas $\mathrm{L}$ mediated induction of hippocampal apoptosis. TNF $\alpha$ mRNA also increased after PCB exposure. Binding of TNF $\alpha$ to TNF R1 results in captor trimerisation and clustering of intracellular death domains [17].

The ligation of Fas to FasL could stimulate NFKB activation [24]. NFkB is a complex of p65 and p50 proteins and is found to be constitutively expressed in mammalian cells. In normal cells, NFKB dimmers are sequestered in the cytoplasm by inhibitory Kappa $\beta$ (IK $\beta$ ). When it is exposed to external signal degradation of IK $\beta$, free NFKB translocates to the nucleus. NFKB exerts both pro and anti-apoptotic effects in testis upon toxicant exposure [25]. Recent study depicted that on hippocampal neurons increased mRNA expressions of NFKB were observed after PCB exposure. This indicates that activation of NFKB is the signaling event that leads to cellular dysfunction and damage. Increased intracellular production of ROS and activated NFKB has been studied in diabetic nephropathic situations also [35]. Induction of NFkB and TNF $\alpha$ after PCB exposure plays a critical role on neurodegeneration.

As discussed earlier, the Fas system has been widely recognized as a key regulator of the apoptosis signal transduction pathways. Fas, is a type 1 transmembrane receptor protein and Fas ligand (Fas L) is a type II transmembrane protein. The binding of FasL to Fas triggers as Fas L mediated apoptotic death in a target cell leading to the activation of caspase 3 . When an external stimulus sensitized to the Fas mediated apoptosis. After PCB exposure induction of both Fas and Fas L levels were observed in rat hippocampus. Increased expression of Fas L in hippocampus triggers apoptotic mechanism suggests that upregulation of Fas L expression in hippocampus and increased activation of caspase 3 may be associated with apoptosis in PCB treated rats. This indicates that $\mathrm{PCB}$ exposure modifies important processes associated with the hippocampal response to $\mathrm{PCB}$ notably downregulating PCB metabolism and increasing apoptosis. Thus it is realistic to show the link between PCB increased ROS production, functional alteration as well apoptosis on hippocampus. It is vulnerable to oxidative stress and PCB toxicity. The damage in other regions such as cerebral cortex and cerebellum is also evident. In our earlier studies also neuronal damages were observed in all the brain regions [16].

\section{ALTERNATIVE DEATH PATHWAYS}

T cells can exert cytotoxicity via exocytosis of their cytotoxic granules [36-38]. The most important components of cytotoxic granules are the pore-forming protein perforin and the lymphocyte-specific granule serine esterase granzyme B (GrB) [39,40]. Perforin and granzymes induce target-cell apoptosis cooperatively. Granzymes trigger caspase activation directly by caspase processing and indirectly by upregulation of proapoptotic genes, whereas perforin supports the appropriate delivery of granzymes by inducing transmembrane pores [38].

\subsection{Perforin/Granzyme Pathway}

T-cell mediated cytotoxicity is a variant of type IV hypersensitivity where sensitized $\mathrm{CD}^{8+}$ cells kill antigenbearing cells. These cytotoxic T lymphocytes (CTLs) are able to kill target cells via the extrinsic pathway and the FasL/FasR interaction is the predominant method of CTL-induced apoptosis [41]. The serine proteases granzyme A and granzyme B are the most important component within the granules [42]. Granzyme B will cleave proteins at aspartate residues and will therefore activate procaspase-10 and can cleave factors like ICAD (Inhibitor of Caspase Activated DNAse) [43]. Reports have also shown that granzyme $\mathrm{B}$ can utilize the mitochondrial pathway for amplification of the death signal by specific cleavage of Bid and induction of cytochrome c release $[44,45]$. However, granzyme $B$ can also directly activate caspase-3. In this way, the upstream signaling pathways are bypassed and there is direct induction of the execution phase of apoptosis.

It is suggested that both the mitochondrial pathway and direct activation of caspase- 3 are critical for granzyme B-induced killing [46]. Earlier studies indicate that this method of granzyme B cytotoxicity is critical as a control mechanism for $\mathrm{T}$ cell expansion of type 2 helper $\mathrm{T}$ (Th2) cells [47]. Moreover, findings indicate that neither death receptors nor caspases are involved with the $T$ cell receptor-induced apoptosis of activated Th2 cells 
because blocking their ligands has no effect on apoptosis. On the other hand, Fas-Fas ligand interaction, adapter proteins with death domains and caspases are all involved in the apoptosis and regulation of cytotoxic type 1 helper cells whereas granzyme B has no effect.

\subsection{Granzymes and Calpain}

$\mathrm{T}$ cells and natural killer (NK) cells utilize a granuleexocytosis pathway for the elimination of virus-infected cells. Cytotoxic granules deliver a pore-forming protein, perforin, and a family of serine proteases known as granzymes into a tightly sealed intercellular synapse, presumably to ensure their selective uptake into target cells. Granzyme B can cleave caspase-3, but also cleaves other substrates including BID and ICAD which results in activation of the CAD DNase as well as alternative pathways of apoptosis $[48,49]$. Granzyme A targets the SET complex resulting in the degradation of selected components, freeing the NM23-H1 DNase and resulting in single-stranded DNA nicks [50]. Granzyme C induces yet another caspase-independent death distinct from either granzyme A or B. Notably, the $\mathrm{Ca}^{2+}$-dependent cysteine protease, calpain also shares some common substrates with the caspases including cleavage of caspases themselves [51].

\subsection{Ischemia Reperfusion}

Occlusion of coronary arteries results in myocardial infarction characterized by massive cell death due to deprivation of oxygen, nutrients and survival factors. Reperfusion of the ischemic tissue is the treatment for acute coronary syndromes and limits the size of the lesion but still results in damage due to oxidative stress, increased cytosolic and mitochondrial calcium levels and inflammation determining what is known as reperfusion injury. Apoptosis plays an essential role in the pathogenesis of ischemia/reperfusion ( $\mathrm{I} / \mathrm{R})$ and in general prolonged ischemia determines an increase in necrosis while reperfusion leads to increased apoptosis. Death during $\mathrm{I} / \mathrm{R}$ is of different kinds: necrotic apoptotic and autophagic. Both the intrinsic and extrinsic apoptotic pathway are involved in I/R. Indeed LPR mice (FAS deficient) show reduced infarct size while deletion of both TNFR1 and TNFR2 appears to have a protective effect. The role of the intrinsic pathway is demonstrated by reduced apoptosis in cardiomyocites over-expressing Bcl-2 or lacking Bax, both in vitro and in vivo. A number of studies have shown that inhibitors of caspases can protect cardiomyocites reducing the infarct size. Interestingly, over-expression of constitutively active Akt or pharmacological sustained activation shows significant protection from apoptosis probably by inactivating a number of proapoptotic proteins [52].

\section{CONCLUSIONS}

An intact death pathway is required for successful embryonic development and the maintenance of normal tissue homeostasis. The deleterious effect of ROS is a function of activation of intracellular cell death circuitry. Involvement of ROS at different phases of the apoptotic pathway, such as induction of mitochondrial permeability transition and release of mitochondrial death amplification factors, activation of intracellular caspases and DNA damage has been clearly established.

The above review showed PCBs induced neuronal apoptosis particularly on hippocampus. PCBs induced oxidative stress and testicular apoptosis have also been studied in our laboratory recently. Understanding the mechanism at its molecular level provided deeper insight into various disease processes and may thus influence therapeutic strategy.

\section{ACKNOWLEDGEMENTS}

The financial assistance to Mr. K. Selvakumar, Senior Research Fellow, Department of Endocrinology from University Grants CommissionResearch Fellowship in Science for meritorious students (UGCRFSMS-SRF) programme and to Dr. J. Arunakaran from, UGC SAP DRS and F. No. 40-374/2011 (SR) (UGC Project), New Delhi are gratefully acknowledged.

\section{REFERENCES}

[1] Okouchi, M., Ekshyyan, O., Maracine, M. and Aw, T.Y. (2007) Neuronal apoptosis in neurodegeneration. Antioxidant and Redox Signaling, 9, 1059-1096. doi:10.1089/ars.2007.1511

[2] Loddick, S.A. and Rothwell, N.J. (1999) Mechanisms of tumor necrosis factor- ${ }^{\circledR}$ action on neurodegeneration: Interaction with insulin-like growth factor-1. Proceedings of National Academy of Sciences USA, 96, 9449-9451. doi:10.1073/pnas.96.17.9449

[3] Safe, S.H. (1994) Polychlorinated biphenyls (PCBs): Environmental impact, biochemical and toxic responses, and implications for risk assessment. Critical Reviews in Toxicology, 24, 87-149. doi:10.3109/10408449409049308

[4] Kamrin, M.A. and Ringer, R.K. (1994) PCB residues in mammals: A review. Toxicology and Environmental Chemistry, 41, 63-84. doi:10.1080/02772249409357961

[5] Mariussen, E. and Fonnum, F. (2001) The effect of polychlorinated biphenyls on the high affinity uptake of the neurotransmitters, dopamine, serotonin, glutamate and GABA, into rat brain synaptosomes. Toxicology, 159, 11-21. doi:10.1016/S0300-483X(00)00374-7

[6] Widholm, J.J., Clarkson, G.B., Strupp, B.J., Crofton, K.M., Seegal, R.F. and Schantz, S.L. (2001) Spatial reversal learning in Aroclor 1254-exposed rats: Sex-specific deficits in associative ability and inhibitory control. Toxicology and Applied Pharmacology, 174, 188-198. 
doi:10.1006/taap.2001.9199

[7] Pravettoni, A., Colciago, A., Negri-Cesi, P., Villa, S. and Celotti, F. (2005) Ontogenetic development, sexual differentiation, and effects of Aroclor 1254 exposure on expression of the arylhydrocarbon receptor and of the arylhydrocarbon receptor nuclear translocator in the rat hypothalamus. Reproductive Toxicology, 20, 521-530. doi:10.1016/j.reprotox.2005.03.008

[8] Engel, R.H. and Evens, A.M. (2006) Oxidative stress and apoptosis: A new treatment paradigm in cancer. Frontiers in Biosciences, 11, 300-312. doi:10.2741/1798

[9] Korrick, S.A. and Sagiv, S.K. (2008) Polychlorinated biphenyls, organochlorine pesticides and neurodevelopment. Current Opinion in Pediatrics, 20, 198-204. doi:10.1097/MOP.0b013e3282f6a4e9

[10] Halliwell, B. and Gutteridge, J. (1999) Free Radicals in Biology and Medicine. Oxford University Press, New York.

[11] Pajović, S.B., Saicić, Z.S., Spasić, M.B. and Petrović, V.M. (2003) The effect of ovarian hormones on antioxidant enzyme activities in the brain of male rats. Physiological Research, 52, 189-194.

[12] Anbalagan, J., Kanagaraj, P., Srinivasan, N., Aruldhas, M.M. and Arunakaran, J. (2003) Effect of polychlorinated biphenyl, Aroclor 1254 on rat epididymis. Indian Journal of Medical Research, 118, 236-242.

[13] Venkataraman, P., Sridhar, M., Dhanammal, S., Vijayababu, M.R., Srinivasan, N. and Arunakaran, J. (2004) Antioxidant role of zinc in PCB (Aroclor 1254) exposed ventral prostate of albino rats. Journal of Nutritional Biochemistry, 15, 608-613. doi:10.1016/j.jnutbio.2004.06.001

[14] Murugesan, P., Kanagaraj, P., Yuvaraj, S., Balasubramanian, K., Aruldhas, M.M. and Arunakaran, J. (2005) The inhibitory effects of polychlorinated biphenyl Aroclor 1254 on Leydig cell LH receptors, steroidogenic enzymes and antioxidant enzymes in adult rats. Reproductive Toxicology, 20, 117-126. doi:10.1016/j.reprotox.2004.11.011

[15] Banudevi, S., Krishnamoorthy, G., Venkataraman, P., Vignesh, R.C., Aruldhas, M.M. and Arunakaran, J. (2006) Role of alpha-tocopherol on antioxidant status in liver, lung and kidney of PCB exposed male albino rats. Food and Chemical Toxicology, 44, 2040-2046. doi:10.1016/j.fct.2006.07.017

[16] Venkataraman, P., Krishnamoorthy, G., Vengatesh, G., Srinivasan, N., Aruldhas, M.M. and Arunakaran, J. (2008) Protective role of melatonin on PCB (Aroclor 1,254) induced oxidative stress and changes in acetylcholine esterase and membrane bound ATPases in cerebellum, cerebral cortex and hippocampus of adult rat brain. International Journal of Developmental Neuroscience, 26, 585-591. doi:10.1016/j.ijdevneu.2008.05.002

[17] Selvakumar, K., Bavithra, S., Suganthi, M., Benson, C.S., Elumalai, P., Arunkumar, R., Krishnamoorthy, G., Venkataraman, P. and Arunakaran, J. (2012) Protective role of quercetin on PCBs-induced oxidative stress and apoptosis in hippocampus of adult rats. Neurochemical Research, 37, 708-721. doi:10.1007/s11064-011-0661-5
[18] Huang, C., Chen, X., Guo, B., Huang, W., Shen, T., Sun, X., Xiao, P. and Zhou, Q. (2012) Induction of apoptosis by icariside II through extrinsic and intrinsic signaling pathways in human breast cancer MCF7 cells. Bioscience Biotechnology and Biochemistry, 76, 1322-1328. doi:10.1271/bbb.120077

[19] Lee, S.H., Shin, M.S., Park, W.S., Kim, S.Y., Kim, H.S., Lee, J.H., Han, S.Y., Lee, H.K., Park, J.Y., Oh, R.R., Jang, J.J., Lee, J.Y. and Yoo, N.J. (1999) Immunohistochemical localization of FAP-1, an inhibitor of Fas-mediated apoptosis, in normal and neoplastic human tissues. Acta Pathologica, Microbiologica et Immunologica Scandinavica, 107, 1101-1108. doi:10.1111/j.1699-0463.1999.tb01515.x

[20] Saradha, B., Vaithinathan, S. and Mathur, P.P. (2009) Lindane induces testicular apoptosis in adult Wistar rats through the involvement of Fas-FasL and mitochondria-dependent pathways. Toxicology, 255, 131-139. doi:10.1016/j.tox.2008.10.016

[21] Krishnamoorthy, G., Selvakumar, K., Elumalai, P., Venkataraman, P. and Arunakaran, J. (2011) Protective role of lycopene on polychlorinated biphenyls (Aroclor 1254)-induced adult rat Sertoli cell dysfunction by increased oxidative stress and endocrine disruption. Biomedicine and Preventive Nutrition, 1, 116-125. doi:10.1016/j.bionut.2011.03.001

[22] Nagata, S. (1999) Fas ligand-induced apoptosis. Annual Reviews in Genetics, 33, 29-55. doi:10.1146/annurev.genet.33.1.29

[23] Hengartner, M.O. and Bryant, J.A. (2005) Apoptotic cell death: From worms to wombats ...but what about the weeds? Symposium on Society of Experimental Biology, 52, 1-12.

[24] Kreuz, S., Siegmund, D., Rumpf, J.J., Samel, D., Leverkus, M., Janssen, O., Häcker, G., Dittrich-Breiholz, O., Kracht, M., Scheurich, P. and Wajant, H. (2004) NFkappaB activation by Fas is mediated through FADD, caspase-8, and RIP and is inhibited by FLIP. Journal of Cell Biology, 166, 369-380. doi:10.1083/jcb.200401036

[25] Kaur, M., Pop, M., Shi, D., Brignone, C. and Grossman, S.R. (2006) hHR23B is required for genotoxic-specific activation of p53 and apoptosis. Oncogene, 26, 12311237. doi: $10.1038 /$ sj.onc. 1209865

[26] Jacobson, M.D. (1997). Programmed cell death: A missing link is found. Trends in Cellular Biology, 7, 467-469. doi:10.1016/S0962-8924(97)01182-3

[27] Mariussen, E. and Fonnum, F. (2006) Neurochemical targets and behavioral effects of organohalogen compounds: An update. Critical Reviews in Toxicology, 36, 253-289. doi:10.1080/10408440500534164

[28] Martin, L.J. (2011) Mitochondrial pathobiology in ALS. Journal of Bioenergetics and Biomembrane, 43, 569-579. doi:10.1007/s10863-011-9395-y

[29] Sánchez-Alonso, J.A., López-Aparicio, P., Recio, M.N. and Pérez-Albarsanz, M.A. (2004) Polychlorinated biphenyl mixtures (Aroclors) induce apoptosis via Bcl-2, Bax and caspase- 3 proteins in neuronal cell cultures. Toxicology Letters, 153, 311-326. doi:10.1016/j.toxlet.2004.05.012 
[30] Mariussen, E., Myhre, O., Reistad, T. and Fonnum, F. (2002) The polychlorinated biphenyl mixture aroclor 1254 induces death of rat cerebellar granule cells: The involvement of the N-methyl-D-aspartate receptor and reactive oxygen species. Toxicology and Applied Pharmacology, 179, 137-144. doi:10.1006/taap.2002.9353

[31] Kass, G.E. and Orrenius, S. (1999) Calcium signaling and cytotoxicity. Environmental and Health Perspectives, 107, 25-35.

[32] Tilson, H.A., Kodavanti, P.R., Mundy, W.R. and Bushnell, P.J. (1998) Neurotoxicity of environmental chemicals and their mechanism of action. Toxicology Letters, 102-103, 631-635. doi:10.1016/S0378-4274(98)00271-9

[33] Baliga, B.C. and Kumar, S. (2002) Role of Bcl-2 family of proteins in malignancy. Journal of Hematology Oncology, 20, 63-74. doi:10.1002/hon.685

[34] Gross, A., McDonnell, J.M. and Korsmeyer, S.J. (1999) BCL-2 family members and the mitochondria in apoptosis. Genes and Development, 13, 1899-1911. doi:10.1101/gad.13.15.1899

[35] Hofmann, K. (1999) The modular nature of apoptotic signaling proteins. Cellular and Molecular Life Sciences, 55, 1113-1128. doi:10.1007/s000180050361

[36] Podack, E.R., Young, J.D. and Cohn, Z.A. (1985) Isolation and biochemical and functional characterization of perforin 1 from cytolytic T-cell granules. Proceeding of National Academy of Science USA, 82, 8629-8633. doi:10.1073/pnas.82.24.8629

[37] Yasukawa, M., Ohminami, H., Arai, J., Kasahara, Y., Ishida, Y. and Fujita, S. (2000) Granule exocytosis, and not the fas/fas ligand system, is the main pathway of cytotoxicity mediated by alloantigen-specific $\mathrm{CD} 4(+)$ as well as $\mathrm{CD} 8(+)$ cytotoxic $\mathrm{T}$ lymphocytes in humans. Blood, 95, 2352-2355.

[38] Trapani, J.A. and Smyth, M.J. (2002) Functional significance of the perforin/granzyme cell death pathway. $\mathrm{Na}$ ture Reviews in Immunology, 2, 735-747.

[39] Nakajima, H., Park, H.L. and Henkart, P.A. (1995) Synergistic roles of granzymes A and B in mediating target cell death by rat basophilic leukemia mast cell tumors also expressing cytolysin/perforin. Journal of Experimental Medicine, 181, 1037-1046. doi:10.1084/jem.181.3.1037

[40] Shi, L., Kam, C.M., Powers, J.C., Aebersold, R. and Greenberg, A.H. (1992) Purification of three cytotoxic lymphocyte granule serine proteases that induce apop- tosis through distinct substrate and target cell interactions. Journal of Experimental Medicine, 176, 1521-1529. doi:10.1084/jem.176.6.1521

[41] Brunner, T., Wasem, C., Torgler, R., Cima, I., Jakob, S. and Corazza, N. (2003) Fas (CD95/Apo-1) ligand regulation in T cell homeostasis, cell-mediated cytotoxicity and immune pathology. Seminars in Immunology, 15, 167176.

\section{doi:10.1016/S1044-5323(03)00035-6}

[42] Pardo, J., Bosque, A., Brehm, R., Wallich, R., Naval, J., Müllbacher, A., Anel, A. and Simon, M.M. (2004) Apoptotic pathways are selectively activated by granzyme A and/or granzyme B in CTL-mediated target cell lysis. Journal of Cell Biology, 167, 457-468. doi: $10.1083 /$ jcb. 200406115

[43] Sakahira, H., Enari, M. and Nagata, S. (1998) Cleavage of CAD inhibitor in CAD activation and DNA degradation during apoptosis. Nature, 391, 96-99. doi:10.1038/34214

[44] Barry, M. and Bleackley, R.C. (2002) Cytotoxic T lymphocytes: All roads lead to death. Nature Reviews in Immunology, 2, 401-409.

[45] Russell, J.H. and Ley, T.J. (2002) Lymphocyte-mediated cytotoxicity. Annual Reviews in Immunology, 20, 323370. doi:10.1146/annurev.immunol.20.100201.131730

[46] Goping, I.S., Barry, M., Liston, P., Sawchuk, T., Constantinescu, G., Michalak, K.M., Shostak, I., Roberts, D.L., Hunter, A.M., Korneluk, R. and Bleackley, R.C. (2003) Granzyme B-induced apoptosis requires both direct caspase activation and relief of caspase inhibition. Immunity, 18, 355-365. doi:10.1016/S1074-7613(03)00032-3

[47] Devadas, S., Das, J., Liu, C., Zhang, L., Roberts, A.I., Pan, Z., Moore, P.A., Das, G. and Shi, Y. (2006) Granzyme B is critical for $\mathrm{T}$ cell receptor-induced cell death of type 2 helper T cells. Immunity, 25, 237-247.

doi:10.1016/j.immuni.2006.06.011

[48] Heusel, J.W., Wesselschmidt, R.L., Shresta, S., Russell, J.H. and Ley, T.J. (1994) Cytotoxic lymphocytes require granzyme B for the rapid induction of DNA fragmentation and apoptosis in allogeneic target cells. Cell, 76, 977-987. doi:10.1016/0092-8674(94)90376-X

[49] Alimonti, J.B., Shi, L., Baijal, P.K. and Greenberg, A.H. (2001) Granzyme B induces BID-mediated cytochrome c release and mitochondrial permeability transition. Journal of Biological Chemistry, 276, 6974-6982. doi:10.1074/jbc.M008444200

[50] Lieberman, J., (2003) The ABCs of granule-mediated cytotoxicity: New weapons in the arsenal. Nature Reviews in Immunology, 3, 361-370. doi:10.1038/nri1083

[51] Gil-Parrado, S., Fernandez-Montalvan, A., Assfalg-Machleidt, I., Popp, O., Bestvater, F., Holloschi, A., Knoch, T.A., Auerswald, E.A., Welsh, K. and Reed, J.C. (2002) Ionomycin-activated calpain kitriggers apoptosis. A probable role for Bcl-2 family members. Journal of Biological Chemistry, 277, 27217-27226. doi:10.1074/jbc.M202945200

[52] Cuadrado, I., Fernandez-Velasco, M., Bosca, L. and de Las Heras, B. (2011) Labdane diterpenes protect against anoxia/reperfusion injury in cardiomyocytes: Involvement of AKT activation. Cell Death and Diseases, 2, e229. doi:10.1038/cddis.2011.113 